platelet engraftment has been known as preferred method for mobilization. Peripheral blood stem cells can be extracted from the autologous or allogeneic donor. Mobilization of the stem cells for autologous stem cell transplant is traditionally done using growth factors alone or in combination with chemotherapy, with or without an additional mobilizing agent. A significant number of hematological malignancy patients are poor mobilizers, (i.e., they are unable to achieve the minimal target cell dose during their first round of mobilization).Therefore, a prediction for a successful stem cell mobilization ideally should be made before initiating any apheresis procedure to spare those with a low rate of success from the risks associated with apheresis procedure. Preapheresis CD34 cell count can predict postapheresis yield and hence, can help to reduce the collection sessions. Reduction of apheresis sessions decreases the discomfort, inconvenience, time, and monetary expenses.

Objectives This study was aimed to analyze preapheresis and postapheresis CD34+ cell counts.

Materials and Methods Patients of any age and gender with diagnosis of hematological malignancies admitted for autologous stem cell transplantation for hematological malignancies (including Hodgkin lymphoma, non-Hodgkin lymphoma, and multiple myeloma) and germ cell tumors in our institute from July 2008 to July 2016 were included in the study. The post-GCSF CBC, preapheresis CBC, CD34+ cell counts, and postapheresis $C B C, C D 34+$ cell counts, mononuclear cell counts to predict the outcome of amount of yield. The effect on engraftment will be measured according to the defining criteria of achieving a sustained peripheral blood neutrophil count of $>500 \times 10^{6} / \mathrm{L}$ (Wolff 2002) and a platelet count of $>20 \times 10^{9} / \mathrm{L}$ (Teltschik et al. 2016) independent of platelet transfusion for at least 7 days. Collection of stem cells was done using apheresis machine (COBE SPECTRA). Complete peripheral blood counts using automated analyzers. Peripheral blood CD34 + cell counts and postapheresis CD34+ cell count using BD FACS CANTO II flow cytometer. To calculate postapheresis yield, the related CD34 count measured by flow cytometer was multiplied by the apheresis product volume and divided by the recipient's body weight $(\mathrm{kg})$. Number of CD34 + cells collected $=($ CD34 cell concentration in final product $) \times($ final product volume).

Results A total of 100 patients who underwent a total of 320 apheresis sessions were included in the study. There were 78 males and 22 females. We also found a significant correlation between preapheresis CD34 + cell count and postapheresis CD34 percentage on days 1, 2 , and 3 of the apheresis sessions. In our study, to obtain more than $1.31 \times 10^{6}$ cells $($ median $=1.04$, range: $0.15-4.70$ ), an absolute count of pre apheresis CD34 + cells $\geq 14$ cells would be necessary. A target of CD34 + cells $\geq 2 \times 10^{6} / \mathrm{kg}$ was obtained in majority of patients if a concentration of $\geq 25 \mathrm{CD} 34$ + cells was present in postapheresis collection. Conclusion Compiling our results with the previous published data, we conclude that there is a strong correlation between preapheresis absolute CD34 + cell counts and postapheresis CD34 + cell count. Our study also suggests that the minimum absolute cell count of $>10$ cells $/ \mu \mathrm{L}$ is required, to achieve a target of $>2-5 \times 10^{6}$ cells for postapheresis yield. Keywords: autologous stem cell transplantation, preapheresis, CD34 + cell count

\section{Epidemiology of Adolescent and Young Adult Cancers in a Tertiary Hospital in South India}

Sahil Gupta', Rasmi Palassery', Santhosh K. Devadas' ${ }^{1}$ Vinayak Maka', Nalini Kilara

'Department of Medical Oncology, M. S. Ramaiah Medical College, Bengaluru, Karnataka, India

Address for correspondence: Sahil Gupta, MBBS, MD (Radiation Oncology), Department of Medical Oncology, M. S. Ramaiah Medical College, Bengaluru 560054, Karnataka, India (e-mail: Dr.sahil1986@ gmail.com).

\section{Abstract}

Introduction There has been an increase in the incidence of malignancies in young Indians, and there is no data reflecting the trend and profile of adolescent and young adult (AYA) cancers.

Objectives This study was aimed to ascertain the epidemiology of AYA cancers in a tertiary care center in south India and the trend of AYA cancers during the past 9 years.
Materials and Methods All patients aged 15 to 39 years with the diagnosis of cancer who were registered and received treatment with M.S. Ramaiah Hospital during a 9-year period from January 2011 to December 2019 were included. Basic demographic information on age, gender was available along with address and contact information. Using cancer site and morphology codes, the cancers were grouped by the ICD-O coding system of AYA cancers and their clinical information on disease and treatment status were collected retrospectively and analyzed.

Results Of the total 946 registered AYA cancer patients, majority of AYA cancer were in age group of 35 to 39 years (39\%) and females (58\%). When analyzing the data and dividing the AYA population into early (15-24 years) and late (25-39 years), we found that whereas the majority of the patients had hematolymphoid malignancies (48\%) in the early group (15-24 years), the late group (25-39 years) had more carcinomas (68\%). The percentage distribution of AYA cancers among the study population, lymphoma and leukemia contribute $11 \%$ and $15 \%$, respectively, to the patient load and still the carcinomas formed the bulk (58\%) of the population. It is interesting to know that breast, genitourinary, and gastrointestinal $(\mathrm{Gl})$ malignancies constituted $17.75 \%, 14.16 \%$, and $14.69 \%$ individually.

Conclusion AYA oncology consists of a heterogeneous population and the profile differs by geography, sex, and other factors. There has been limited improvement in the past decade but there is a lot more to be done. To assess the problem, we have to identify and characterize the problem and look at the epidemiology of this population. This will require multicenter and international studies with focus on improving outcomes as in pediatric inspired ALL protocols. The trials should be started at local levels to ensure maximum participation. We need to generate data on epidemiology and channel our resources properly to save this precious but so called lost tribe of oncology.

Keywords: AYA oncology, India, epidemiology

\section{Weekly Etoposide and Platinum in Small-Cell Lung Cancer: Hope and Scope for Fragile Patients}

Deepak Sundriyal' ${ }^{1}$, Parmod Kumar ${ }^{1}$, Yeswanth Gogisetti ${ }^{2}$, Amit Sehrawat ${ }^{1}$

'Department of Medical Oncology, Hematology, All India Institute of Medical Sciences, Rishikesh, Uttrakhand, India

2Department of Medicine, All India Institute of Medical Sciences,

Rishikesh, Uttrakhand, India

\section{Address for correspondence:}

Dr Deepak Sundriyal MD, DNB Department of Medical

Oncology AlIMS, Rishikesh, Dehradoon 249203, India

(e-mail: drdeepaksundriyal@gmail.com).

\section{Abstract}

Introduction Small-cell lung cancer (SCLC) is an aggressive and chemo-sensitive disease. Many patients present in an advanced stage and with a poor performance status (PS). In such a condition, the treatment dilemma due to poor condition advocates alternative treatment approach rather than standard chemotherapy. One way of usual practice is to split the chemotherapy into a weekly schedule. However, there is limited data regarding the actual benefit of weekly chemotherapy. We hypothesize that a weekly chemotherapy with etoposide/platinum combination will be feasible and safe in patients of advanced-stage SCLC with poor PS.

Objectives This study was aimed to determine whether weekly etoposide/platinum chemotherapy is a safe option for patients with advanced stage, poor PS, and SCLC who are otherwise unfit for systemic anticancer therapy.

Materials and Methods We retrospectively analyzed the data of SCLC patients presented to our center from July 2018 to September 2020 . We analyzed that treatment, survival, and clinical benefit data. We also analyzed the benefit of weekly etoposide/platinum in otherwise unfit for chemotherapy.

Results One hundred and fifty patients of lung cancer presented to our department between July 2018 and September 2020; SCLC constituted $34 \%$ (53 cases). In SCLC patients, the median overall survival was 2.5 months. Fourteen (26\%) patients with SCLC were unable to start any oncological intervention. Ten (19\%) patients could receive 